\title{
Ethical Considerations of Patient-Funded Research for Multiple Sclerosis Therapeutics
}

\author{
Lilyana Amezcua $^{1}$ (D) . Flavia Nelson ${ }^{2}$
}

Published online: 4 August 2017

(C) The American Society for Experimental NeuroTherapeutics, Inc. 2017

\begin{abstract}
Patient-funded research has started to emerge in multiple sclerosis studies, such as low-dose naltrexone and stem-cell therapy. While these represent greater opportunities for the physician, scientist, and patient, ethical concerns concerning protocol review, conflict of interests, and protection of subjects are reviewed.
\end{abstract}

Keywords Multiple sclerosis $\cdot$ Ethics $\cdot$ Patient-funded research

\section{Introduction}

Multiple sclerosis (MS) is now considered one of the most expensive diseases in neurology, with the recognition that once officially diagnosed the individual will be on diseasemodifying therapy the rest of their life [1]. An important issue is the recognition that $>10$ phase III clinical trials which occurred in the last 10 years have been successful in bringing new therapeutics to market and in diversifying the care of MS. Part of this success has been attributed to multiple initiatives set forth by nonprofit organizations culminating in Food and Drug Administration (FDA) fast-track approval.

Electronic supplementary material The online version of this article (doi:10.1007/s13311-017-0560-9) contains supplementary material, which is available to authorized users.

Lilyana Amezcua

lamezcua@usc.edu

1 Department of Neurology, Keck School of Medicine, University of Southern California, Los Angeles, CA, USA

2 Department of Neurology, McGovern Medical School, University of Texas, Houston, TX, USA
Patient-funded clinical research is a contemporary approach that is becoming mainstream, with the goal of accelerating and translating investigations to clinical care [2-4]. The term "translating" in the medical research context means to transform findings from bench to bedside or bedside to community, with the goal of achieving meaningful outcomes. Patient-funded research involves patients or organized patient groups who directly fund research that is not being fully funded by public entities or industry sponsors. This is distinct from pay-to-participate trials or "pay to play", which involves patients who pay to enroll in research studies or pay for experimental treatments and/or interventions where principles of social value and fair subject selection have been question in the past [5]. In the case of patient-funded research, the patient is empowered to take on a advocacy role to procure research/ trial in a field or treatment of interest where they might directly benefit. While it could be viewed as altruistic and philanthropic in nature, recent reports suggest that patient-funded trials are not necessarily free of ethical concerns. In fact, patientfunded projects may be more prone to inadequate peer-review platforms, they may pose a higher threat to high-quality scientific evidence $[6,7]$, and are not free of conflict of interests (COIs). While these are common concerns across all clinical trials, these threats appear to be increasing in patient-funded research studies.

Only a few patient-funded studies in MS have been officially published $[8,9]$. However, over the years there has been an increase in organizations financially supported by patients dedicated to accelerating MS research (i.e., The Accelerated Cure Project for Multiple Sclerosis is a patient-funded national nonprofit organization dedicated to accelerating advances toward a cure for MS). While this new approach should be welcomed, there exists a level of warning concerning future rigor and conduct of studies. All studies, patient funded or not, should be carefully designed, rigorously justified, and 
properly conducted in order to protect the rights, interests, and welfare of patients and contribute to generalizable scientific knowledge [10].

In the present article, we highlight potential risks and benefits of patient-funded research and discuss COIs and vulnerable populations as standard principles of pragmatic clinical trials. We also suggest strategies for rigorous study design and study conduct within strict ethical boundaries, in order to optimally engage in future patient-funded research in MS.

\section{Low-Dose Naltrexone as a Patient-Funded Study}

A contemporary clinical trial in MS that was published as "supported by private contributions" was a study by Cree et al. [8], which was conducted at a major academic institution. It was a single-center, double-masked, placebo-controlled, crossover study that evaluated the efficacy of treatment with $4.5 \mathrm{mg}$ nightly naltrexone [low-dose naltrexone (LDN)] over 8 weeks. In the study by Cree et al. [8], the primary outcome was set to evaluate changes in selfreported quality of life in patients with MS. The study reported a high dropout rate, with $25 \%$ of enrolled individuals not completing the study. Management errors were also reported. The authors concluded that these 2 factors led to a reduction in the trial's statistical power. In this setting, one could question if the trial was well planned, if there was proper institutional review board (IRB) oversight, and if enough attention was placed on sample size estimations [11]. Nevertheless, a systematic evaluation of LDN to confrim efficacy was needed.

\section{An Ethical Response to Patients' Need}

To conduct a study that has potential to improve the clinical management of MS could be viewed as an ethical response to patients' needs. The LDN study can be viewed as having had the potential to generalize the results to the target population. Generating data and delivering outcomes more rapidly was also of potential benefit. In addition, capitalization on the potential success of the trial might have provided support for academic advancement of the clinician-scientist, which is an inherent need and could be viewed as a COI. Nevertheless, the trial, although not successful, highlighted the longstanding ethical principles that require that risks to research participants be minimized and justified by the value of the knowledge the study was expected to produce, and the feasibility of extending the knowledge to other populations that would benefit from it.

\section{Patient-Funded Stem Cell Trials and Issues}

Numerous recent clinical studies conducting stem-cell therapy in MS have emerged [12-18]. The discovery of highly multipotent stem-cell types and alternative methods of isolating, creating, and using them brings increased benefits both to the patient and scientist/clinician. However, patient-funded clinical trials using stem-cell base therapy should raise questions and concerns [19]. Outside of institutional oversight, issues of consent, control, and justice should not be taken lightly.

In 2013, the FDA approved autologous, mesenchymal stem cell-derived neural progenitor cells as an Investigational New Drug for a phase I clinical trial in the treatment of MS at the Tisch MS Research Center of New York.

"To my knowledge, this is the first FDA-approved stem cell trial in the United States to investigate direct injection of stem cells into the cerebrospinal fluid of MS patients, and represents an exciting advance in MS research and treatment", said Dr. Saud A. Sadiq, Senior Research Scientist at Tisch MS Research Center of New York and the study's principal investigator.

The study investigated a regenerative strategy using stem cells harvested from the patient's own bone marrow. The stem cells were injected intrathecally. Preclinical testing found that injection of these cells may decrease brain inflammation and promote myelin repair and/or neuroprotection [20,21]. The study results were presented at scientific meetings reporting statistically significant improvements in disability scores in some patients with progressive MS, with a promise for a future, larger study to confirm the results.

This primary stem cell-based patient-funded study has had a national reach to patients, which has promoted the opportunity to further procure funds for the next phase II trial. Anecdotal reports suggest that patients with MS have reached the principal investigator to request the treatment outside a trial, under the FDA's compassionate rule. Clinicianscientists in many states were also approached by patients with MS and families about the possibility to become a site for the future phase II trial and/or starting a trial at their particular institution.

The fact that some of these trials, such as the example above, have proven beneficial has created a certain degree of pressure on clinician-scientists who have the ability and interest to conduct clinical trials. According to citations from a 2015 article in the national post "As funding becomes harder and harder to secure for all the good work that legitimate clinical trials seek to accomplish, society needs to be open to this evolving creative process. Patient-funded trials can also close a gap that major pharmaceutical companies are unwilling to fill, testing stem cells and other products that are difficult to patent - and therefore unlikely to attract huge sums of money for traditional testing". 
Overall, one could assume that well-conducted patientfunded clinical trials are a win-win situation for patients, clinicians, and the healthcare system, but the ethical concerns behind the so-called "pay to participate" trials are a reminder that patient-funded research could potentially run the risk of either being highly or poorly regulated by IRBs/Committee for the Protection of Human Subjects (CPHS) or even being prohibited at major universities in order to avoid significant COIs with philanthropists, donors, and funded clinical trials (see Table 1).

\section{Recommendations}

The trial at Tish in New York undeniably caused excitement and anxiety for many patients and physicians who engaged in multiple discussions that can be viewed as exhausting and time consuming. Considerations to improve communication would be to acknowledge the scientific advancement in the field, as well as community interest by way of newsletters, a website, or research update lectures and reporting accurate results with an interpretation by the clinician(s) at that MS center, including limitations of the study in a way that patients can understand it.

The possibilities of conducting similar trials at that particular institution should be addressed at an individual

Table 1 Case scenario

EG is a 39-year-old man with relapsing-remitting multiple sclerosis (MS) and significant disability. He has been deteriorating over time despite trying multiple disease-modifying treatments. Recently he has become interested in the advances in stem-cell transplants. While discussing the difficulties of obtaining funding for these kind of studies with his MS specialist, EG proposes to donate and organize a fundraiser for stem-cell transplant research at the university's MS center, where his clinical care takes place, "as long as I get a spot in the trial".

Questions

Is it ethical for the institution to receive funding with conditions? Is there potential harm in the physician promising a spot in the research to his patient?

Patient-clinician relationship becomes a delicate issue when the donor expects a certain degree of "access" to the study he/she is interested in funding and or expects "special treatment" because of it. This becomes more problematic when the clinician proposes a randomized, placebo-controlled, double-blind study, which can potentially place the donor in the placebo arm. Other issues such as the expectation of being informed of the progress of the planning phases or expecting the physician to consider suggestions and opinions can also become an issue with important donors. Another consideration is the actual knowledge of the donors/patients and the sources from which they are obtaining this information, which can result in unrealistic expectations not only for outcomes, but also for the process of adequately designing and conducting a trial and the length of time the complete process may take.

"There is no good evidence to support the empirical assumption that research studies, as a class, are more likely than clinical practice to run counter to the medical best interests of patients" (Hastings Center Report 2013 [23]). institutional level. Our goal should be to recognize that we want to improve neurological disability in those affected with MS while withstanding the pressure from patients to make irrational decisions and have unrealistic expectations of what a clinician-scientist may be able to accomplish.

\section{Ethical Considerations in Patient-Funded Research}

As previously mentioned, patient-funded research studies can create a certain number of issues and other pressures on the clinician scientist and the academic institution conducting the trial that are not necessarily dealt with or seen with other clinical trials such as industry sponsored trials.

\section{Scientific Integrity and Study Design}

Clinician-scientists must create the study protocol with the rigor of study that could be submitted for funding to a government agency. Studies should not be designed to obtain the desired outcome; in particular, they should not be designed to benefit important donors, despite the pressure they may assert. Ideally, the protocol would be designed before donors are approached. Donors may specifically ask for studies to be designed to benefit them or at least ensure inclusion. The investigator would have to decide between the ideal study or a less ideal but "feasible to be funded" study that would also benefit the local MS community. Donors may be uncomfortable with studies that would have a placebo control arm, which is ideal when evaluating a new therapeutic strategy. This can be managed by using an established treatment for MS instead of placebo.

\section{Time to Enrollment}

Donors may not understand the time necessary for protocol design, recruitment of necessary personnel and approval by a local IRB. Establishing realistic expectations from the planning phases as well as the use of official updates may be extremely beneficial in maintaining a sense of teamwork and inclusion without allowing interference from donors.

\section{Selection Bias and Social Justice}

Inclusion/exclusion criteria should be carefully defined to maximize the potential efficacy of the experimental therapy to be tested so as to include patients with MS who are most likely to benefit by it, with emphasis on not tailoring the criteria so as to allow inclusion of all donors. The potential of being excluded from the trial should be carefully explained to donors prior to obtaining the funds, emphasizing the benefit of the information gained by the study should the donor be excluded. 


\section{Access}

Ideally, enrollment should be open to all patients in a community, but it is understandable that patients from a particular MS center would have priority for enrollment, especially if the center is supporting the study financially. However, this may pose a selection bias, which would then need to be addressed. Discussions regarding this issue should happen during the planning phases, especially when considering that patientfunded research would likely continue to be limited to studies with small number of patients, as most MS research studies can have a high cost, and donations in general cannot compete with industry or government agencies, with some exceptions.

\section{Dissemination of Results to the Scientific Community}

It is important to maintain an engagement with the rest of the scientific community via scientific presentations at conferences, publications, and peer-to-peer communication so that the MS community may benefit from learning the outcomes, whether positive or negative.

\section{Best Practice Guidelines}

Best practice guidelines can be used to infer the ethical and scientific standards in conducting the specified clinical research (Table 2). In addition, those involved in patientfunded research should create educational material explaining the study; this should preferably be written at a 5 to 6 grade level and with pictures/graphics to further explain the concepts, including a time frame and time line for each step of the study and when results are expected. This material should be tested for accuracy and clarity, prior to IRB approval and discussed with patients in detail once they have had the opportunity to see it. It can be done as a video, website, meeting with handouts or pamphlets, but not as a pure discussion, as it

Table 2 Best-practice considerations

1. Is the intent of the project either to test a novel hypothesis or to replicate another researcher's original study?

2. Will patients or personnel be exposed to additional discernable risks or burdens beyond those of usual care at the institution?

3. Does the project involve withholding of any aspect of conventional care shown to be beneficial in prior well-conducted clinical trials?

4. Does the project seek to test interventions, practices, or treatments that are not standard of care (neither consensus-based nor evidence-based)?

5. Will the safety and/or effectiveness of a drug or regulated device be evaluated or be compared with that of another?

6. Will the project be described as research in grants, public presentations, academic dossier, or other representations?

7. Does the project have funding from an organization with a commercial interest in the use of the results? is easy to assume the information presented was understood when it was not.

Informed consent should include a line stating that the patient has read the additional information and currently has no further questions. A questionnaire with key questions of issues that could cause confusion can also be given to each patient and reviewed by the investigator prior to the consenting process. Considerations regarding $\mathrm{COI}$ and vulnerable populations are also important.

\section{COIs}

A COI occurs when an individual who is involved in multiple interests has one interest that might interfere with another. A COI arises mostly out of investigators' relation with the sponsor and perceived financial COIs to the study or care are a threat to trust. The now more common COI arises from the emergence of physician payment as salaried-supported investigators in industry-sponsored trials where the physician recruitment practices may be stirred away from the patient's best interests [20]. In the case of patient-funded clinical trials, the investigator is likely to have a relationship with the patient, where that patient or patients may be largely motivated by seeing the medication, product, or tool be accessible in a shorter time. In addition to patient-related conflicts, the institution itself may itself view patient-funded research as a revenue source given that research overhead is likely to be higher for patient-funded trials than for National Institutes of Health-based.

The US Department of Health and Human Services recognizes that some conflicting financial interests in research may affect the rights and welfare of human subjects [9]. In a recent study that examined if physician compensation for pharmasponsored clinical trial in MS influences patient trust, it was found that much of the trust differences were due to individuals past experience with being in a clinical trial. Nevertheless, mistrust was seen by both groups of individuals if salary support was provided by the sponsor (38-61\%) [24]. This highlights the importance of communication with patients concerning COIs. The institutional ethics committees and investigator both have the responsibility of identifying such conflicts and to ensure that these do not compromise the protection of human subjects and the outcome of the trial.

According to Pham-Kanter et al. [25] "Patients' contact with physicians who receive industry payments is more prevalent than physician-based measures of industry contact would suggest. Very few Americans know whether their own doctor has received industry payments or are aware that payment information is publicly available". This becomes especially important when clinicians who receive industry payments become involved in patient-funded research especially if the "drug" to be tested is related to stem cells. A case in point is a private company called Celltex Therapeutics 
Corporation; the company has not only attempted to establish a relationship with clinician-scientists in academic centers in the area, but has also asked those clinicians to agree to participate in data collection with financial compensation for patients undergoing paid transplants outside the trial. Some of these relationships are necessary in order to complete these trials, but the clinician-scientist is encouraged to minimize the role as advisor under compensation once the trial in question is approved by the IRB.

\section{Recommendations}

COI exists independently of whether decisions are affected by a personal interest. A COI can be viewed as the potential for bias to creep into the conduct of research, not the likelihood that bias actually is present. Disclosure of COIs by the clinician-scientist and the Sunshine Act help in promoting rigorous ethical standards and maintenance should be required in patient-funded research [26]. This should be part of the discussion that takes place before the consent is given; a signature space attesting to the above should also be included in the consent form. In the case of a clinician-scientist with COIs and inability to eliminate them before conducting the trial, the COI should be submitted to an ethics committee that will determine the conditions under which the clinician may continue to engage in paid compensation by the company causing the COI with the clinical trial. These so-called COI management plans are reviewed on an annual basis to ensure that conditions have not changed and reassess the possibility of biases affecting the trial's outcomes.

Much of the COIs that exemplify the Sunshine Act reports refer to tangible COIs; those that are measurable and quantifiable. The issue of intangible COIs need to also be considered. These involve personal, prejudicial, and professional conflicts that pertain to growth, institutional aspirations, and personal opinions. Because the nature of patient-funded research will usually involve a personal relationship that exists between the community base organization and/or patient and physician running the trial, the potential for intangible COIs should be carefully examined. Devising new strategies to manage, reduce, or eliminate intangible and tangible COIs is expected to be an ongoing challenge [27].

\section{Vulnerable and Special Populations}

There are ethical considerations in research that should be considered where the possibility of social factors is likely to intersect with health care. The concept of who is considered vulnerable is used in research ethics to signify those identified as needing extra protection over and above the usual protection conferred to those who participate in research. More commonly, a vulnerable and/or special population can be an

individual who is economically disadvantaged, a minority, pregnant, has children, or has a chronic health condition, including cognitive and mental illness (Table 3) [28-31]. Other special populations include students and employees, where participation may be perceived to be required to prevent discrimination and/or loss of benefits [31]. In MS, vulnerable populations are encountered in our day-to-day practice and the integration into clinical trials to provide some level of care in those with less access can be tempting. In MS, potential vulnerable populations include minority groups such as African Americans, Hispanics (primarily Spanish-speaking), and children. While it is no longer morally or medically acceptable to exclude them, it is important to consider the established guidelines and regulations set forth by the federal government under the Common Rule [32], to ensure voluntariness and freedom from undue influence such as coercion, manipulation, or persuasion. Vulnerable populations deserve special procedural protections as they have been the victims of unethical behavior in research in the past.

While no specific publish examples exist in the MS literature, it is important to recognize that in the recent years minority populations are now of interest to clinical trial participation [33]. Hispanics and African Americans with MS are poorly understood and have been reported to lack adequate knowledge of MS [34], and there is the possibility that patientfunded research would seek to fund projects dedicated to this subject. It will be important to recognize that patient-funded research that primarily seeks to fund a project that involves a vulnerable group has the potential to be high impact and provide the greatest potential for improved health outcomes. Also, ethical concerns would lie in the recruitment, selection process, and ability to conduct culturally competent research. The inclusion of minorities would benefit from community engagement, which could ensure sensitive approaches and potential delivery of more effective interventions that are specific to that populations need.

Ethical concerns also exist in the conduct and feasibility of pediatric trials in MS [35], with a recent emphasis on examining if adult treatments in MS have the same efficacy or risks in children. The recognition that children are vulnerable individuals because they do not have the autonomy to decide whether to participate in a trial or to refuse participation requires we deliver clear communication and develop a strong

Table 3 Vulnerable and special populations
Children and minors

Pregnant

Prisoners

Cognitive and mentally ill

Minorities

Terminally ill

Students 
commitment with the parent or guardian. Lastly, women with MS are increasingly contemplating reproductive life and the treatment potential at this stage exist [36].

\section{Recommendations}

Patient-funded clinical research suffers from the same vulnerabilities that can be driven by power to recruit into a study. We need to be cognizant of the past research that has taken place in minority populations - this includes understanding the short- and long-term consequences that trials such as the Tuskegee Study in syphilis in African Americans brought to the community. Understand that patients, participating or funding, may be identified as vulnerable which could still place them at risk. Thus, clinical investigators need to be aware of the federal regulations, the challenges they may impose, and be able to report and protect them in their review under their IRB.

\section{Conclusion}

We recognize that patient-supported research is likely to increase in the MS field as experimental treatment options continue to be considered. Our job as clinician-scientists will not only be to provide our patients with education governing participation, but will also necessitate the alliance with a third body to examine our own COIs in order to maintain the highest possible scientific quality while addressing the patient needs to be involved in the process. Pharma and governments may not be willing to pay for the studies necessary to improve patient care, and payers are unlikely to support experimental treatments that have not been tested or approved by the FDA, in which case it leaves payment/sponsorship in the hands of patients, increasing the potential for ethical dilemmas.

Acknowledgements Expanded Access (Compassionate Use). Available at www.fda.gov/NewsEvents/PublicHealthFocus/ ExpandedAccessCompassionateUse/. Accessed April 4, 2017. Financial conflict of interest: HHS Guidance. Available at www.hhs.gov/ohrp/ regulations-and-policy/guidance/financial-conflict-of-interest/ Accessed April 4, 2017

\section{References}

1. Hartung DM, Bourdette DN, Ahmed SM, Whitham RH. The cost of multiple sclerosis drugs in the US and the pharmaceutical industry: too big to fail? Neurology 2015;84:2185-2192.

2. Vayena E, Brownsword R, Edwards SJ, et al. Research led by participants: a new social contract for a new kind of research. J Med Ethics 2016;42:216-219.

3. Vayena E, Tasioulas J. The ethics of participant-led biomedical research. Nat Biotechnol 2013;31:786-787.
4. Giannuzzi V, Devlieger H, Margari L, et al. The ethical framework for performing research with rare inherited neurometabolic disease patients. Eur J Pediatr 2017;176:395-405.

5. Lind SE. Fee-for-service research. N Engl J Med 1986;314:312-315.

6. Wenner DM, Kimmelman J, London AJ. Patient-funded trials: Opportunity or liability? Cell Stem Cell 2015;17:135-137.

7. Vayena E, Tasioulas J. Adapting standards: ethical oversight of participant-led health research. PLOS Med 2013;10:e1001402.

8. Cree BA, Kornyeyeva E, Goodin DS. Pilot trial of low-dose naltrexone and quality of life in multiple sclerosis. Ann Neurol 2010;68:145-150.

9. Perry JE, Cox D, Cox AD. Trust and transparency: patient perceptions of physicians' financial relationships with pharmaceutical companies. J Law Med Ethics 2014;42:475-491.

10. Emanuel EJ, Wendler D, Grady C. What makes clinical research ethical? JAMA 2000;283:2701-2711.

11. Sakpal TV. Sample size estimation in clinical trial. Perspect Clin Res 2010;1:67-69.

12. Connick P, Kolappan M, Crawley C, et al. Autologous mesenchymal stem cells for the treatment of secondary progressive multiple sclerosis: an open-label phase 2a proof-of-concept study. Lancet Neurol 2012;11:150-156.

13. Mancardi GL, Sormani MP, Gualandi F, et al. Autologous hematopoietic stem cell transplantation in multiple sclerosis: a phase II trial. Neurology 2015;84:981-988.

14. Nash RA, Hutton GJ, Racke MK, et al. High-dose immunosuppressive therapy and autologous hematopoietic cell transplantation for relapsing-remitting multiple sclerosis (HALT-MS): a 3-year interim report. JAMA Neurol 2015;72:159-169.

15. Harris VK, Vyshkina T, Sadiq SA. Clinical safety of intrathecal administration of mesenchymal stromal cell-derived neural progenitors in multiple sclerosis. Cytotherapy 2016;18:1476-1482.

16. Douvaras P, Wang J, Zimmer M, et al. Efficient generation of myelinating oligodendrocytes from primary progressive multiple sclerosis patients by induced pluripotent stem cells. Stem Cell Rep 2014;3:250-259.

17. Burt RK, Loh Y, Cohen B, et al. Autologous non-myeloablative haemopoietic stem cell transplantation in relapsing-remitting multiple sclerosis: a phase I/II study. Lancet Neurol 2009;8:244-253.

18. Freedman MS, Cohen JA. Meta-analysis of bone marrow transplantation treatment studies: mixing 'apples and oranges'. Mult Scler 2011;17:131-132.

19. Simonson OE, Domogatskaya A, Volchkov P, Rodin S. The safety of human pluripotent stem cells in clinical treatment. Ann Med 2015;47:370-380.

20. Weinfurt KP, Friedman JY, Allsbrook JS, Dinan MA, Hall MA, Sugarman J. Views of potential research participants on financial conflicts of interest: barriers and opportunities for effective disclosure. J Gen Intern Med 2006;21:901-906.

21. Harris VK, Yan QJ, Vyshkina T, Sahabi S, Liu X, Sadiq SA. Clinical and pathological effects of intrathecal injection of mesenchymal stem cell-derived neural progenitors in an experimental model of multiple sclerosis. J Neurol Sci 2012;313:167-177.

22. http://nationalpost.com/health/trend-sees-patients-paying-for-theirown-clinical-trials-but-critics-raiseethical-concerns/wcm/ da5a7fb6-59c8-4cdb-afa4-d5a90c590776. Accessed July 29, 2017

23. http://www.thehastingscenter.org/wp-content/uploads/2013annual-report.pdf.

24. Klein E, Solomon AJ, Corboy J, Bernat J. Physician compensation for industry-sponsored clinical trials in multiple sclerosis influences patient trust. Mult Scler Relat Disord 2016;8:4-8.

25. Pham-Kanter G, Mello MM, Lehmann LS, Campbell EG, Carpenter D. Public awareness of and contact with physicians who receive industry payments: a national survey. J Gen Intern Med 2017;32:767-774. 
26. Corboy JR. The relationship between physicians and Pharma: playing the devil's advocate. Neurol Clin Pract 2014;4:161-163.

27. Spelsberg A, Martiny A, Schoenhoefer PS. Is disclosure of potential conflicts of interest in medicine and public health sufficient to increase transparency and decrease corruption? J Epidemiol Commun Health 2009;63:603-605.

28. Kastner B, Behre S, Lutz N, et al. Clinical research in vulnerable populations: variability and focus of institutional review boards' responses. PLOS ONE 2015;10:e0135997.

29. Gehring K, Sitskoorn MM, Aaronson NK, Taphoorn MJ. Interventions for cognitive deficits in adults with brain tumours. Lancet Neurol 2008;7:548-560.

30. 28. Ramsay RE, Rowan AJ, Pryor FM. Special considerations in treating the elderly patient with epilepsy. Neurology 2004;62(5 Suppl. 2):S24-S29.
31. Schwenzer KJ. Protecting vulnerable subjects in clinical research: children, pregnant women, prisoners, and employees. Respir Care 2008;53:1342-1349.

32. Menikoff J, Kaneshiro J, Pritchard I. The common rule, updated. N Engl J Med 2017;376:613-615.

33. Khan O, Williams MJ, Amezcua L, Javed A, Larsen KE, Smrtka JM. Multiple sclerosis in US minority populations: clinical practice insights. Neurol Clin Pract 2015;5:132-142.

34. Shabas D, Heffner M. Multiple sclerosis management for lowincome minorities. Mult Scler 2005;11:635-640.

35. Tenembaum SN. Ethical challenges in paediatric clinical trials in multiple sclerosis. Ther Adv Neurol Disord 2012;5:139-146.

36. Morgan-Followell BN, Nicholas JA, Weisleder P. Reproductive issues in women with multiple sclerosis: ethical considerations. Continuum (Minneap Minn) 2014;20:177-180. 